\title{
Fully developed laminar buoyant flow in vertical cavities and ducts of bounded section
}

\author{
By G. D. McBAIN \\ School of Engineering, James Cook University, Townsville, Queensland 4811 Australia
}

(Received 29 May 1998 and in revised form 12 August 1999)

The fully developed flow in a vertical cavity or duct subject to horizontal heating is considered. Solutions of the Boussinesq equations are obtained for rectangular and elliptic sections, in terms of Fourier series and polynomials, respectively. Both generalize the familiar odd-symmetric cubic profile of the plane cavity. Uniqueness is demonstrated under the restriction that the flow is independent of height. For cavities with rectangular sections, it is predicted and verified that the flow in the plane of spanwise symmetry is practically independent of the span if this exceeds 1.7 times the breadth.

\section{Introduction}

In 1954 Batchelor investigated the flow in a fluid-filled cuboid with one opposing pair of vertical walls $(x= \pm b / 2)$ at temperatures $T=T_{0} \pm \Delta T / 2$. His attention was restricted to the limiting case of infinite spanwise aspect ratio, $\mathscr{S}$ (see figure $1 a$ ); the velocity and temperature were assumed to be independent of $z$, the spanwise coordinate. He realized that if the cavity was narrow enough; i.e. $b$ small enough or $\mathscr{A}$, the vertical aspect ratio, large enough; a fully developed region might exist in which the one-dimensional solution of Jones \& Furry (1946) would apply. That is, the temperature would vary linearly between the hot and cold walls and the purely vertical velocity would have an odd-symmetric cubic profile. Eckert \& Carlson (1961) named this the 'conduction regime', and observed its existence in a cavity of finite $\mathscr{A}$.

Here, we consider a different two-dimensional approximation of the cuboid: $\mathscr{A} \rightarrow \infty$ with $\mathscr{S}$ arbitrary, so that the velocity and temperature are now functions only of $x$ and $z$. The vertical variation of pressure is retained due to the hydrostatic contribution. The solution for this system provides an answer to the question of how large $\mathscr{S}$ must be for the flow to be adequately approximated by a two-dimensional model in the conduction regime.

Implicit here is the assumption that the length scale of the flow in any direction is the same as the corresponding dimension of the cavity, so that we are restricted to unicellular flows. This will be the case if $b$ (and therefore the Grashof number, $G r \equiv g \beta \Delta T b^{3} / v^{2}$, where $g$ is the gravitational field strength, $\beta$ the thermal coefficient of volumetric expansion and $v$ the kinematic viscosity) is small enough, since then the viscous layers adjacent the hot and cold walls will extend across the entire breadth.

The one-dimensional solution of Jones \& Furry (1946) obviously cannot apply near the floor and ceiling of the cavity since there the flow must be turned, but Batchelor (1954) estimated that the conduction regime would not be disrupted by the penetration of convective effects from the ends into the core unless the Rayleigh number, $R a$ ( $\equiv G r v / \alpha$, where $\alpha$ is the thermometric conductivity), was greater than 
$500 \mathscr{A}$, though various other values have been suggested: 1000 by Elder (1965); 3000 by Vest \& Arpaci (1969) and Gebhart et al. (1988, p. 730); 316 $\mathscr{A}$ by Gill \& Davey (1969); 1500 by Dixon \& Probert (1975) and 645 $\mathscr{A}$ for infinite Prandtl number, $\operatorname{Pr}(\equiv v / \alpha)$, by Daniels (1985). All of these criteria are based on the two-dimensional limit, $\mathscr{S} \rightarrow \infty$.

Gill \& Davey (1969) pointed out that for tall air-filled cavities the conduction regime would be destroyed by instability rather than the gradual effect described above. For Prandtl numbers less than 10 they gave a critical Grashof number of 8030, which is the value appropriate for the fluid contained between two infinite plane walls held at different temperatures (Gershuni \& Zhukhovitskii 1976, ch. 10). An investigation of the three-dimensional stability of the conduction regime flow is beyond the scope of the present paper, but since the flow only weakens with decreasing $\mathscr{S}$ (see below), it is expected that the critical Grashof number for instability in any cavity of bounded section would be larger. This would be analogous to the stability of the fluid in finite cuboids heated from below; Davis (1967) found that as the length of the rolls, i.e. the span, decreased from infinity, the critical Rayleigh number increased. Thus, while the conduction regime is somewhat restrictive-Gill \& Davey (1969) calculated that the critical temperature difference for a $1 \mathrm{~cm}$ air gap is around $40 \mathrm{~K}$, but only $5 \mathrm{~K}$ for a $2 \mathrm{~cm}$ gap-it may be slightly less so once the third dimension is taken into account.

The importance of treating tall cavities as three-dimensional has been stressed by Chait \& Korpela (1989) for another reason. They showed that for air in the $\mathscr{A}, \mathscr{S} \rightarrow \infty$ case, the secondary two-dimensional multiple cells are only stable up to $G r=8550$, beyond which the flow is no longer confined to vertical-transverse planes.

There have been several numerical treatments of the flow in vertical cuboids, the special case of the cube $(\mathscr{A}=\mathscr{S}=1)$ being recently proposed as a benchmark computational fluid dynamics problem (Leong, Hollands \& Brunger 1998, 1999). Most studies have dealt with values of $\mathscr{A}$ near unity (e.g. Viskanta, Kim \& Gau 1986; Mallinson 1987; Fusegi, Hyun \& Kuwahara 1993). This is probably because the greatest accuracy for a given number of grid points is achieved if the solution domain is a cube, according to Mallinson \& de Vahl Davis (1977), who did treat cases with $\mathscr{A}=5$, but only for a very few values of the Grashof number all of which were higher than 10000 . Batchelor's two-dimensional criterion indicates that a fully developed flow could only be expected in a cavity of this height if the Grashof number were less than about 2500, and indeed, the effects of the horizontal surfaces were felt throughout the height of the cavity. Thus, our search of the literature has uncovered no numerical investigations of the effect of the endwalls on the conduction regime.

In the Handbook of Heat Transfer Fundamentals, Raithby \& Hollands (1985) state that very little information exists on the effect of $\mathscr{S}$ on the overall heat transfer rate for moderate to high $\mathscr{A}$. This paper supplies one part of the answer to this complicated question.

\section{General model}

Consider the steady laminar motion set up in a diathermanous Newtonian fluid by buoyancy forces due to temperature variations. Under the Boussinesq approximation, i.e. the effect of thermal expansion is limited to a linear buoyancy force, the fluid properties are otherwise constant and viscous dissipation of energy is negligible, the 
(a)

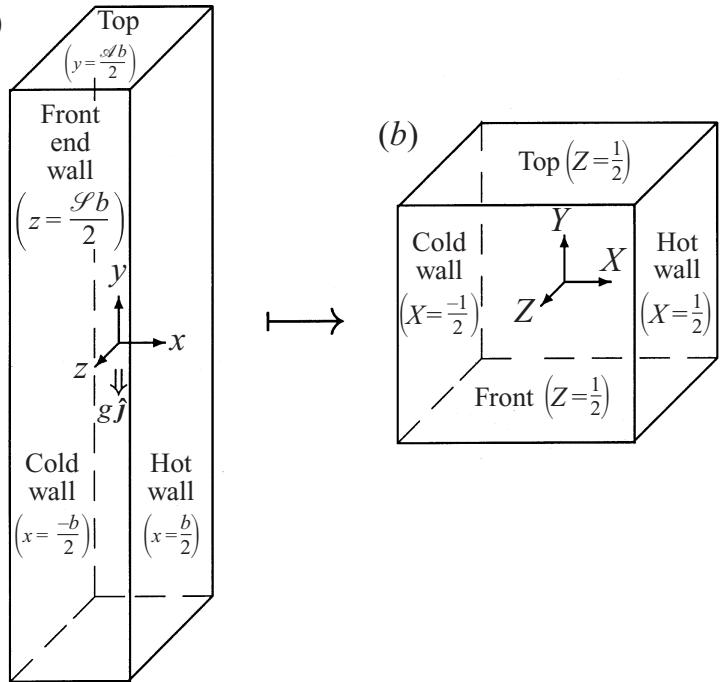

FIGURE 1. Geometry for cavities and ducts of rectangular section in $(a)$ primitive and (b) non-dimensional coordinates.

field equations are (Mallinson \& de Vahl Davis 1973)

subject to

$$
\begin{gathered}
\boldsymbol{u} \cdot \nabla T=\alpha \nabla^{2} T, \\
\boldsymbol{u} \cdot \nabla \boldsymbol{u}=-\nabla\left(\frac{p}{\rho}+g y\right)+g \beta\left(T-T_{0}\right) \hat{\boldsymbol{j}}+v \nabla^{2} \boldsymbol{u},
\end{gathered}
$$

$$
\nabla \cdot \boldsymbol{u}=0
$$

where $p$ and $\rho$ are the pressure and density, and the $y$-direction, with unit vector $\hat{\boldsymbol{j}}$, has been chosen as vertical (see figure 1).

At the walls,

$$
\boldsymbol{u}=0 .
$$

A general thermal boundary condition is

$$
C\left(\frac{T-T_{0}}{\Delta T}-\frac{x}{b}\right)+I\left(\frac{b}{\Delta T} \hat{\boldsymbol{n}} \cdot \nabla T-\hat{\boldsymbol{n}} \cdot \hat{\boldsymbol{\imath}}\right)=0,
$$

where $\hat{\boldsymbol{n}}$ is the unit outward normal, $\hat{\boldsymbol{\imath}}$ is the unit vector in the $x$-direction, normal to the hot and cold wall (see figure 1), $\Delta T$ is a temperature difference defined by this relation and $C$ and $I$ are dimensionless scalars defined on the walls, which may vary with position so long as they do not both vanish at any point. If $C \equiv 0, T$ is rendered determinate by

$$
\iiint T \mathrm{~d} x \mathrm{~d} y \mathrm{~d} z=T_{0},
$$

where the integral extends over the entire domain.

On the endwalls, $I=0$ leads to a linear temperature variation, while $C=0$ gives an adiabatic condition. On the heated and cooled walls, $C=0$ implies a uniform heat flux while $I=0$ gives an isothermal condition. If the solid presumed to be surrounding the fluid has a higher thermal conductivity, $I=0$ everywhere is a simple and consistent idealization (Batchelor 1954), although the condition $I=0$ on the 
heated and cooled walls and $C=0$ on the connecting walls appears frequently in the literature (Mallinson \& de Vahl Davis 1973, 1977; Schladow, Patterson \& Street 1989; Fusegi et al. 1991).

\section{The large- $\mathscr{A}$ limit}

A solution is sought for infinitely tall or narrow spaces; the method is essentially similar to that used by McBain (1998) for vapour transport through a gas-filled layer bounded by parallel vertical walls. The equations are non-dimensionalized and the domain is transformed to a cube (see figure 1). This moves the geometric parameters, $\mathscr{A}$ and $\mathscr{S}$, into the field equations. This is meaningful if the scales of the motion correspond to those of the space in the same direction, and so not, for example, if multicellular flow prevails (see $\S 1$ ).

The dimensionless field variables are

$$
\begin{gathered}
\boldsymbol{U} \equiv U \hat{\boldsymbol{i}}+V \hat{\boldsymbol{j}}+W \hat{\boldsymbol{k}} \equiv \boldsymbol{U}_{\perp}+V \hat{\boldsymbol{j}} \equiv \frac{v \boldsymbol{u}}{g \beta \Delta T b^{2}}, \\
P \equiv \frac{p+\rho g y}{\mathscr{A} \rho g \beta \Delta T b}, \\
\theta \equiv \frac{T-T_{0}}{\Delta T},
\end{gathered}
$$

and the new coordinates are $X \equiv x / b, Y \equiv y / \mathscr{A} b$ and $Z \equiv z / \mathscr{S} b$ as illustrated in figure $1(b)$.

In the limit $\mathscr{A} \rightarrow \infty$, with $G r, \operatorname{Pr}$ and $\mathscr{S}$ fixed and finite, the equations reduce to

$$
\begin{gathered}
G r \operatorname{Pr} \boldsymbol{U}_{\perp} \cdot \nabla_{\perp} \theta=\nabla_{\perp}^{2} \theta, \\
\operatorname{Gr} \boldsymbol{U}_{\perp} \cdot \nabla_{\perp} V=-\frac{\partial P}{\partial Y}+\theta+\nabla_{\perp}^{2} V, \\
\nabla_{\perp} P=0,
\end{gathered}
$$

subject to

$$
\nabla_{\perp} \cdot U_{\perp}=0 \quad \text { in } \Omega^{\square}
$$

and

$$
\begin{gathered}
U=V=W=0 \quad \text { on } \partial \Omega^{\square}, \\
C(\theta-X)+I \hat{\boldsymbol{n}} \cdot\left(\nabla_{\perp} \theta-\hat{\imath}\right)=0 \quad \text { on } \partial \Omega^{\square},
\end{gathered}
$$

where $\nabla_{\perp}$ is a horizontal gradient operator:

$$
\nabla_{\perp} \equiv \frac{\partial}{\partial X} \hat{\boldsymbol{\imath}}+\frac{1}{\mathscr{S}} \frac{\partial}{\partial Z} \hat{\boldsymbol{k}}
$$

$\Omega^{\square}$ is the domain $\{\mathbf{X}:|X|<1 / 2,|Z|<1 / 2\}$, and $\partial \Omega^{\square}$ is its boundary.

There should also be boundary conditions at $Y= \pm 1 / 2$, corresponding to the entrance and exit of the duct or the ceiling and floor of the cavity, but these cannot be met by the solution of equations (3.4)-(3.7). The problem is singular in the sense of perturbation theory, as the basic solution cannot be uniformly valid over the domain. For very large $\mathscr{A}$, however, the region of non-uniformity should be limited to thin layers near $Y= \pm 1 / 2$, corresponding to a small proportion of the unscaled cavity in terms of $y$. The full set of equations (2.1)-(2.3) would have to be solved to obtain the flow in these regions. This restriction is an advantage for the present work, though, as 
it means that the same fully developed solution will apply to both ducts and cavities. Some distinction between these will be made in $\S 3.3$.

Since the horizontal components of the momentum equation (2.2) have degenerated to (3.6), a statement of the horizontal uniformity of the pressure, $U$ and $W$ are determined only by the continuity equation (3.7) and the boundary conditions. An obvious solution is

$$
U=W=0 .
$$

Accepting this solution (uniqueness follows from Theorem 1, proven in the Appendix, if the velocity is assumed to be independent of $Y$ ) the heat equation (3.4) reduces to

$$
\nabla_{\perp}^{2} \theta=0 .
$$

The unique solution satisfying this and the boundary condition (3.9), for all values of $C$ and $I$, is

$$
\theta=X
$$

Substituting this into the vertical momentum equation leads to

$$
\nabla_{\perp}^{2} V=\frac{\mathrm{d} P}{\mathrm{~d} Y}-X,
$$

subject to $V=0$ on $X= \pm 1 / 2$ and $Z= \pm 1 / 2$. This is conveniently decomposed by defining the forced and natural contributions, $V_{f}$ and $V_{n}$, satisfying

$$
\begin{gathered}
V_{f}+V_{n}=V, \\
\nabla_{\perp}^{2} V_{f}=\frac{\mathrm{d} P}{\mathrm{~d} Y}, \\
\nabla_{\perp}^{2} V_{n}=-X,
\end{gathered}
$$

and

$$
V_{f}=V_{n}=0 \quad \text { on } \partial \Omega^{\square} .
$$

\subsection{Forced flow}

The equation (3.16) for $V_{f}$ is simply that for fully developed laminar flow in a duct of rectangular section, the solution for which is well known (Dryden, Murnaghan \& Bateman 1956, p. 197):

$$
\frac{V_{f}}{-\mathrm{d} P / \mathrm{d} Y}=\frac{V_{f}^{\|}}{-\mathrm{d} P / \mathrm{d} Y}-4 \sum_{k=0}^{\infty} \frac{(-1)^{k}}{[(2 k+1) \pi]^{3}} \cos [(2 k+1) \pi X] \frac{\cosh [(2 k+1) \pi \mathscr{S} Z]}{\cosh [(2 k+1) \pi \mathscr{S} / 2]},
$$

where $V_{f}^{\|}$is the solution valid for $\mathscr{S} \rightarrow \infty$ (Lamb 1932, p. 582):

$$
\frac{V_{f}^{\|}}{-\mathrm{d} P / \mathrm{d} Y}=\frac{1-4 X^{2}}{8} \text {. }
$$

\subsection{Natural flow}

Solution of (3.17) requires a particular integral; two rational choices are the asymptotic solutions for large and small $\mathscr{S}$.

The solution valid in the limit $\mathscr{S} \rightarrow \infty$ is

$$
V_{n}^{\|}=\frac{X\left(1-4 X^{2}\right)}{24} .
$$


This result, due to Jones \& Furry (1946), and used by Batchelor (1954) and many others (Vest \& Arpaci 1969; Hart 1971; Gershuni \& Zhukhovitskii 1976; Nagata \& Busse 1983; Daniels 1985; Chait \& Korpela 1989) in the analysis of tall cavities, describes the flow between infinite parallel plane vertical walls. It satisfies (3.18) at $X= \pm 1 / 2$ but not at $Z= \pm 1 / 2$. The combination $V_{f}^{\|}+V_{n}^{\|}$was noted by Aung (1972) as the solution for the transversely heated duct problem with $\mathscr{S} \rightarrow \infty$.

The solution of equation (3.17) matching the boundary conditions (3.18) then follows readily by Fourier's method:

$$
V_{n}=V_{n}^{\|}+\frac{1}{4 \pi^{3}} \sum_{k=1}^{\infty} \frac{(-1)^{k}}{k^{3}} \sin (2 k \pi X) \frac{\cosh (2 k \pi \mathscr{S} Z)}{\cosh (k \pi \mathscr{S})} .
$$

The difference $V_{n}-V_{n}^{\|}$quantifies the effect of the endwalls.

For small $\mathscr{S}$, the geometry approaches that of Hele-Shaw's experiments (Lamb 1932 , p. 582)-the dominant effect on the flow being the viscous damping from the endwalls. The limiting form of the vertical component of velocity is

$$
V_{n}^{=}=\mathscr{S}^{2} \frac{X\left(1-4 Z^{2}\right)}{8} .
$$

This function satisfies the boundary conditions (3.18) at $Z= \pm 1 / 2$, but not those at $X= \pm 1 / 2$. The corresponding full solution is

$$
V_{n}=V_{n}^{=}-\frac{2 S^{2}}{\pi^{3}} \sum_{k=0}^{\infty} \frac{(-1)^{k}}{(2 k+1)^{3}} \frac{\sinh [(2 k+1) \pi X / \mathscr{S}]}{\sinh [(2 k+1) \pi / 2 \mathscr{S}]} \cos [(2 k+1) \pi Z] .
$$

The difference $V_{n}-V_{n}^{=}$quantifies the effect of the hot and cold walls on the Hele-Shaw flow.

Both (3.22) and (3.24) are exact solutions of (3.17) and (3.18), and also, when combined with (3.13), (3.11) and (3.19), the full equation of motion (2.2), for all values of $\mathscr{S} \in(0, \infty)$.

\subsection{Completely enclosed flows}

Since $V_{n}$ is odd in $X$, its integral over the section is zero and the only contribution to a net vertical flow rate comes from $V_{f}$. If the physical system of finite $\mathscr{A}$, modelled by equations (3.4)-(3.9) when $\mathscr{A}$ is large, has solid surfaces at $y= \pm b \mathscr{A} / 2$, the net vertical flow rate must vanish (to conserve mass) so that

$$
\begin{gathered}
\frac{\mathrm{d} P}{\mathrm{~d} Y}=0, \\
V_{f}(X, Z)=0,
\end{gathered}
$$

and

$$
V(X, Z)=V_{n}(X, Z)
$$

A similar line of reasoning leads to the conclusion that $\mathrm{d} P / \mathrm{d} Y$ is uniform in the case of a duct, where the planes $Y= \pm 1 / 2$ now represent orifices. Indeed, $\mathrm{d} P / \mathrm{d} Y$ is proportional to the net vertical flow rate (Dryden et al.1956, p. 197).

Contours of $V_{n}$, calculated using (3.22), are shown in figure 2, using the unstretched coordinates $x$ and $z$. The curves can be interpreted as either contours of $V_{n}$ or vortex-lines, since the vertical gradient of velocity is zero (see Theorem 2 in the Appendix). 


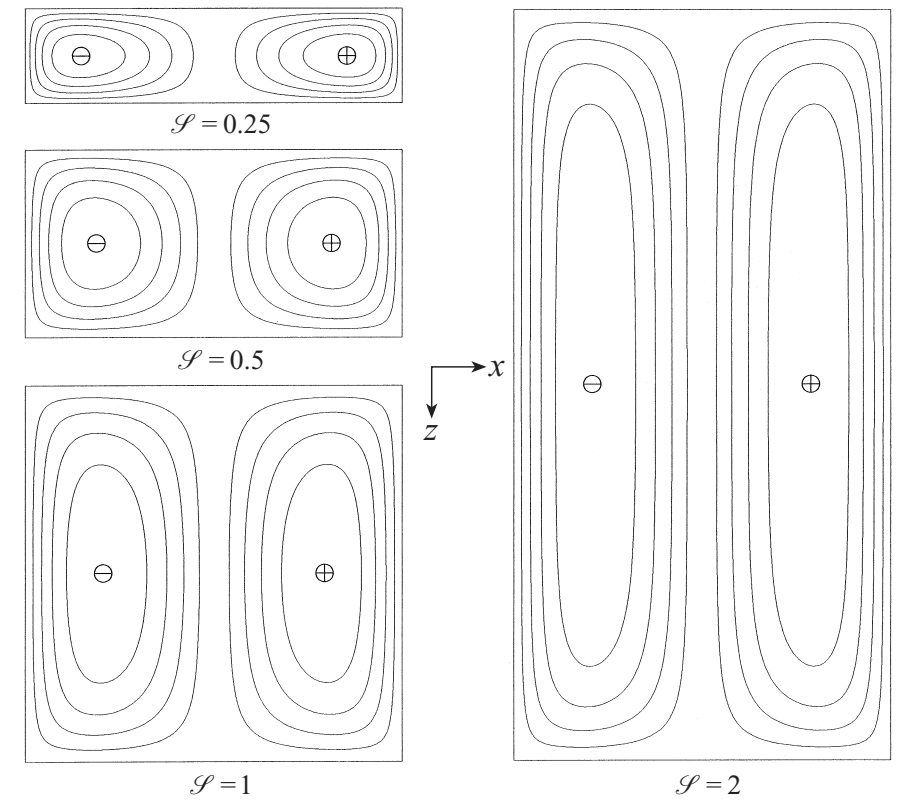

FIGURE 2. Fully developed buoyancy-induced flow in various rectangular sections. Curves can be interpreted as either vortex-lines or contours of the vertical component of velocity with levels at $\pm 20(20) 80 \%$ of the maximum. $\oplus$ and $\ominus$ mark the extrema of $V_{n}$ (points of zero vorticity).

\section{Extent of the effect of the endwalls}

The solutions (3.22) and (3.24) are difficult to evaluate numerically for very large or small values of $\mathscr{S}$. There is a physical reason for this: each of the two shorter vertical walls (when $\mathscr{S}$ is far from unity) affects only a small (roughly square) portion of the section, so that each should be sensibly independent of the other's presence. The solutions (3.22) and (3.24), however, combine the effects of the two.

The solution for $V_{n}$ (3.22) can be re-expressed:

$$
V_{n}=V_{n}^{\sqcup}+\frac{1}{4 \pi^{3}} \sum_{k=1}^{\infty} \frac{(-1)^{k}}{k^{3}} \sin (2 k \pi X) \frac{\mathrm{e}^{-k \pi \mathscr{S}}\left[\mathrm{e}^{-2 k \pi \mathscr{S}|Z|}-\mathrm{e}^{-2 k \pi \mathscr{S}(1-|Z|)}\right]}{1-\mathrm{e}^{-2 k \pi} \mathscr{S}}
$$

where

$$
V_{n}^{\sqcup}=V_{n}^{\|}+\frac{1}{4 \pi^{3}} \sum_{k=1}^{\infty} \frac{(-1)^{k}}{k^{3}} \sin (2 k \pi X) \exp [-k \pi \mathscr{S}(1-2|Z|)] .
$$

This is now a full solution that is practical for large $\mathscr{S}$ in the vicinity of either the front or back endwall. For $Z>0$, (4.2) gives the effect of the front wall; with $|Z|$ replaced by $Z$, it would be the solution of (3.17) in the semi-infinite rectangular section $\{(X, Z):-1 / 2<X<1 / 2, Z<1 / 2\}$. Thus, (4.1) separates the effects of the two endwalls that were entangled in (3.22). An alternative expression for (3.24) can be derived in an entirely analogous way.

The representation (4.1) of $V_{n}$ suggests a means of finding a simple approximate answer to one of the principal questions of this study: how large $\mathscr{S}$ must be for the flow in the spanwise plane of symmetry to be essentially described by the Jones-Furry solution (3.21). Near the front endwall $(Z=1 / 2)$ it is clear that $V_{n}-V_{n}^{\sqcup}$ can be neglected in comparison to $V_{n}^{\sqcup}$ if $\mathscr{S}$ is sufficiently large. The effect of the $k$ th term 

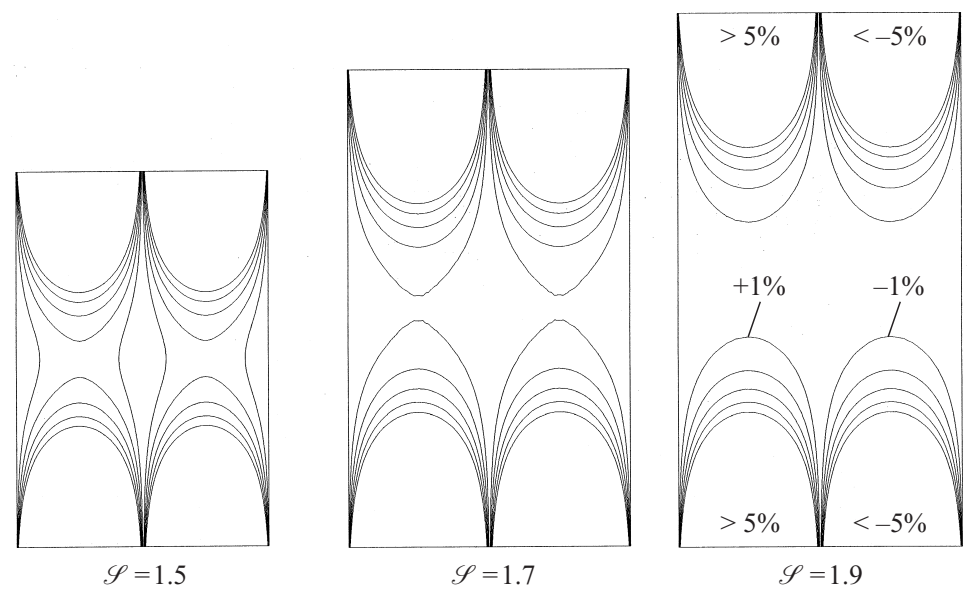

FIGURE 3. The effect of the endwalls. Contours of the difference between the full solution (4.1) and the Jones-Furry limiting form (3.21). Contours at $\pm 1(1) 5 \%$ of the maximum $\left(=\max V_{n}^{\|}=\sqrt{3} / 216\right)$, as labelled in the rightmost plot.

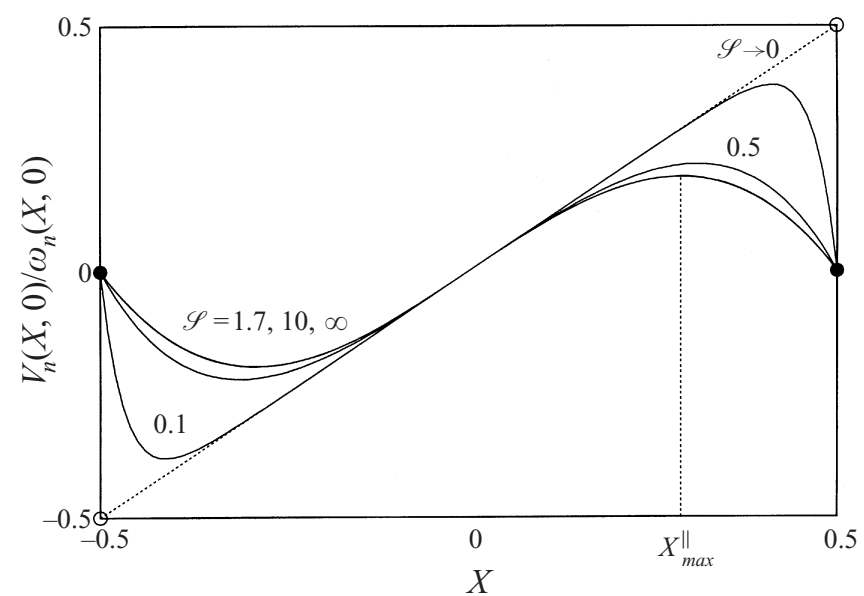

FIGURE 4. Buoyancy-induced velocity, $V_{n}$, in the plane of spanwise symmetry, $Z=0$, of a duct or cavity of rectangular section, normalized by the magnitude of the vorticity, $\omega_{n} \equiv \partial V_{n} / \partial X$, at the section centre.

of the series in (4.2) has reduced in magnitude by a factor of e after a distance (in the scale of $Z$ ) from the front endwall of $1 /(2 k \pi \mathscr{S})$. Thus the first term, which also has the largest magnitude due to the factor $k^{-3}$, acts over the longest distance. It has reduced to less than $0.5 \%$ of its influence at $Z=1 / 2$ for

$$
Z \leqslant \frac{1}{2}-\frac{\ln 200}{2 \pi \mathscr{S}}
$$

Since the location of the plane of spanwise symmetry is $Z=0$, we may expect the flow there to be sensibly independent of the presence of the endwalls for $\mathscr{S} \geqslant \ln (200) /$ $\pi \doteq 1.7$. Applying an analogous procedure to $V_{f}$ leads to $\mathscr{S} \geqslant 2 \ln (200) / \pi \doteq 3.4$.

To investigate this approximation, plots of the difference between the actual solution for $V_{n}$, calculated from (4.1) for each half of the section, and the Jones-Furry solution (3.21) are given in figure 3 for values of $\mathscr{S}$ spanning the estimate $\mathscr{S}=1.7$. The figure 

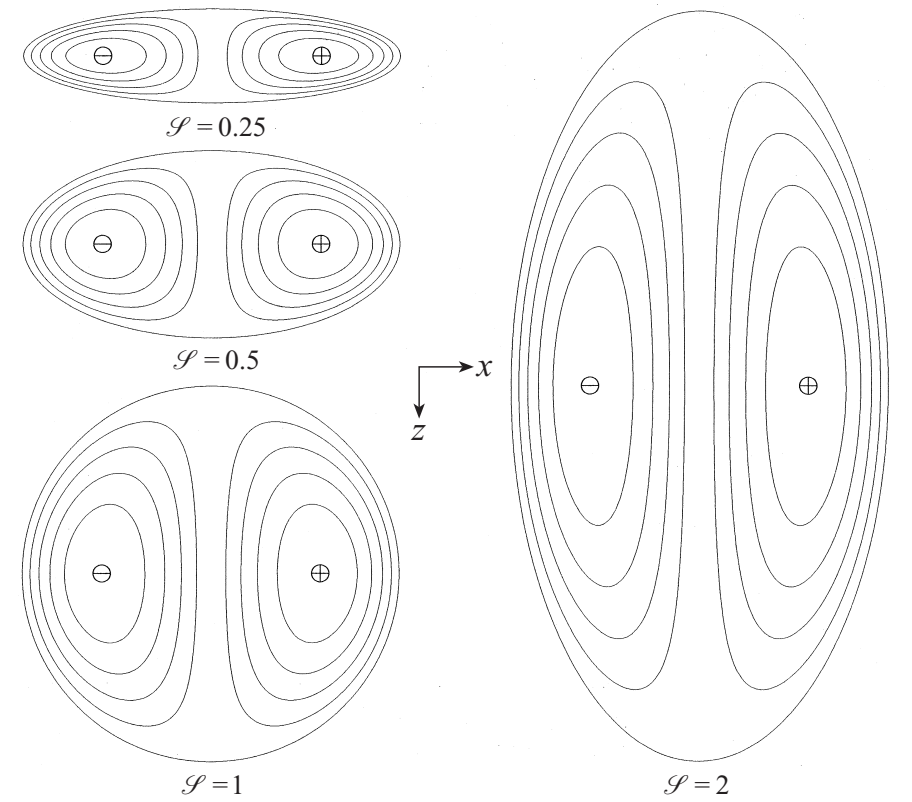

FIGURE 5. Fully developed buoyancy-induced flow in various elliptic sections. Curves can be interpreted as either vortex-lines or contours of the vertical component of velocity with levels at $\pm 20(20) 80 \%$ of the maximum. $\oplus$ and $\ominus$ mark the extrema of $V_{n}$ (points of zero vorticity).

shows that the departure from the two-dimensional Jones-Furry limit is indeed less than $1 \%$ for $\mathscr{S} \geqslant 1.7$.

The least effect of the endwalls is felt in the plane of spanwise symmetry. Velocity profiles there are plotted in figure 4 . The endwalls are seen to distort the flow, as well as weaken it: the maximum of velocity moves from $X_{\max }^{\|}=\sqrt{3} / 6$ in the Jones-Furry limit, $\mathscr{S} \rightarrow \infty$, toward $X=1 / 2$ as $\mathscr{S} \rightarrow 0$. Figure 4 again confirms that for $\mathscr{S}>1.7$, the flow in the plane $Z=0$ is essentially two-dimensional.

\section{Sections other than rectangular}

Solutions for cavities and ducts with sections other than rectangular can readily be obtained by similar means. Here we present details for one: the elliptic section with semiaxes $b / 2$ and $\mathscr{S} b / 2$, defined by

$$
\Omega^{\circ}=\left\{X: X^{2}+Z^{2}<1 / 4\right\} .
$$

The forced flow solution (Lamb 1932, p. 587) is

$$
\frac{V_{f}^{\circ}(X, Z)}{-\mathrm{d} P / \mathrm{d} Y}=\frac{1-4\left(X^{2}+Z^{2}\right)}{8\left(1+\mathscr{S}^{-2}\right)},
$$

and the natural flow is given by

$$
V_{n}^{\circ}(X, Z)=\frac{X\left[1-4\left(X^{2}+Z^{2}\right)\right]}{8\left(3+\mathscr{S}^{-2}\right)},
$$

of which the special case $\mathscr{S}=1$ (circular section) was reported by Ostroumov (1958). It is also similar to the fully developed flow in a long axially heated tube (Klosse \& 
Ullersma 1973; Bejan \& Tien 1978). Contours of $V_{n}^{\circ}$ are displayed for various values of $\mathscr{S}$ in figure 5. Again, the curves can alternatively be interpreted as vortex-lines.

Apart from the simplicity of the result (5.3), the elliptic section is remarkable for two reasons. First, the velocity profile in the plane $Z=0$ has the same odd-symmetric cubic shape as the Jones-Furry flow (3.21) for all values of $\mathscr{S}$, only the amplitude varies (being within $1 \%$ of the large $\mathscr{S}$ asymptote for $\mathscr{S}>\sqrt{33} \doteq 5.7$ ). The second is the comparative ease with which the thermal boundary conditions (3.9) may be imposed. Consider the cavity or duct to be surrounded by a highly conducting solid in which, at large distances, the temperature gradient is uniform and parallel to the $x$-axis. The problem of the temperature distribution in the solid is analogous to that for potential flow relative to an elliptic cylinder moving uniformly along the axis. From the solution to the latter problem (Lamb 1932, p. 84), it can be seen that the temperature at the section boundary varies linearly with $x$, i.e. (3.9) applies with $I=0$. This example may be a reasonable model of the air-filled holes in some bricks or masonry blocks used in building walls, the thermal conductivity of a typical concrete being about fifty times that of air.

\section{Conclusion}

The flow in a transversely heated vertical cavity or duct of bounded horizontal section was considered in the limit as the vertical scale of motion tends to infinity. Solutions were presented for rectangular and elliptic sections.

The temperature in all cases varies linearly with the transverse coordinate. The expressions for the purely vertical velocity in rectangular and elliptic sections are infinite hyperbolic-trigonometric series and bivariate polynomials of degree 3 , respectively. These match all the conditions of the full problem for vertical cavities or ducts of finite height except that the boundary conditions at the top and bottom and are independent of the Grashof and Prandtl numbers, provided that the Grashof number is small enough for the flow to be stable. How the critical Grashof number changes as the spanwise aspect ratio decreases from infinity (the two-dimensional limit) is an important but as yet unanswered question, though it is suggested that it can only increase.

Aside from the applicability of the 'general' model, the basic assumptions of the analysis are: (i) the appropriateness of the fairly general, but still idealized, thermal boundary conditions; (ii) that $\mathscr{A}$ is sufficiently large for a fully developed region to exist; (iii) that $G r$ is sufficiently small for the flows presented to be stable.

For large sectional spans, the flows approach the familiar odd cubic profile, which also exists in the plane of spanwise symmetry of an elliptic section of arbitrary $\mathscr{S}$. In the rectangular section, the velocity profile for $Z=0$ is distorted as $\mathscr{S}$ decreases, with the location of the extrema moving outward from $X= \pm \sqrt{3} / 6$ toward the hot and cold walls; for $\mathscr{S} \geqslant 1.7$, however, the profile and magnitude are practically independent of $\mathscr{S}$.

Other properties of the solution, and the extension to simultaneous heat and mass transfer, are discussed in the author's thesis (McBain, forthcoming).

This work forms part of an Australian Research Council supported project. The author would like to thank Dr Jonathan Harris for making available accurate finite element solutions, which proved very illuminating in the development of this work, and, along with Dr Harry Suehrcke and Professor Don Close, for frequent helpful discussion. 
Appendix. Two theorems on fully developed flow

THEOREM 1 (UNIDIRECTIONALITY OF FULLY DEVELOPED FLOW). Let $\boldsymbol{U}$ be a regular stationary solution of the incompressible Navier-Stokes equations with a purely vertical body force. If $\boldsymbol{U}$ vanishes on the boundary of a vertically prismatic domain and has zero vertical gradient then $\boldsymbol{U}$ is purely vertical.

Proof. If $\hat{\boldsymbol{j}}$ is the unit vertical vector, we have $\hat{\boldsymbol{j}} \cdot \nabla \boldsymbol{U}=0$ and the equation of continuity reduces to

$$
\nabla_{\perp} \cdot U_{\perp}=0 .
$$

The horizontal components of the equation of motion become

$$
\operatorname{Re} \boldsymbol{U}_{\perp} \cdot \nabla_{\perp} \boldsymbol{U}_{\perp}=-\nabla_{\perp} p+\nabla_{\perp}^{2} \boldsymbol{U}_{\perp}
$$

where $R e$ is the Reynolds number. The remnant of the equation of continuity (A 1) implies the existence of a scalar function, $\psi$ (see Lamb 1932, p. 62 for a construction) such that

$$
\begin{gathered}
U=-\frac{1}{\mathscr{S}} \frac{\partial \psi}{\partial Z} \\
W=\frac{\partial \psi}{\partial X},
\end{gathered}
$$

so that the horizontal momentum problem becomes

$$
\frac{\operatorname{Re}}{\mathscr{S}}\left(\frac{\partial \psi}{\partial Z} \frac{\partial}{\partial X}-\frac{\partial \psi}{\partial X} \frac{\partial}{\partial Z}\right) \nabla_{\perp}^{2} \psi=-\nabla_{\perp}^{4} \psi
$$

subject to

$$
\psi=\frac{\partial \psi}{\partial n}=0 \quad \text { on } \partial \Omega
$$

where $\partial / \partial n$ is the outward normal derivative. On multiplying (A 5) through by $\varphi$, an arbitrary function also satisfying the boundary conditions (A 6), and integrating over the section, the variational form is obtained (Girault \& Raviart 1979, p. 120):

$$
\frac{R e}{\mathscr{S}} \iint_{\Omega} \nabla_{\perp}^{2} \psi\left[\frac{\partial \psi}{\partial Z} \frac{\partial \varphi}{\partial X}-\frac{\partial \psi}{\partial X} \frac{\partial \varphi}{\partial Z}\right] \mathrm{d} X \mathscr{S} \mathrm{d} Z=\iint_{\Omega}\left(\nabla_{\perp}^{2} \psi\right)\left(\nabla_{\perp}^{2} \varphi\right) \mathrm{d} X \mathscr{S} \mathrm{d} Z .
$$

If $\varphi$ is then set equal to $\psi$, the integrand on the left-hand side vanishes and the equation reduces to

$$
\iint_{\Omega}\left(\nabla_{\perp}^{2} \psi\right)^{2} \mathrm{~d} X \mathscr{S} \mathrm{d} Z=0
$$

which is only possible if $\nabla_{\perp}^{2} \psi \equiv 0$. With the boundary conditions (A 6), and the wellknown uniqueness of harmonic functions in bounded domains (Lamb 1932, pp. 41, 64), we have $\psi=0$ throughout $\Omega$ and so $U=W=0$, uniquely.

Note: The horizontal components of velocity satisfy a two-dimensional NavierStokes problem with no body force and homogeneous boundary conditions. The integral (A 8) is proportional to the net rate of viscous dissipation of energy by this horizontal flow per unit length in the vertical direction (Lamb 1932, p. 580). Under the hypotheses there is no source for this power. Thus, the entire proof is similar to that of the uniqueness of creeping flows with prescribed boundary velocities (Lamb 1932, p. 617), even though inertia was not neglected here. 
THEOREM 2. If a flow has zero gradient in some direction, the component of velocity in this direction is constant along vortex-lines.

Proof. The hypothesis may be written

$$
\hat{\boldsymbol{e}} \cdot \nabla \boldsymbol{u}=0 .
$$

Adding three terms (the first and third of which are identically zero due to the uniformity of $\hat{\boldsymbol{e}}$ ) to each side of the hypothesis and forming the scalar product with the vorticity gives

$$
(\nabla \times \boldsymbol{u}) \cdot[\hat{\boldsymbol{e}} \cdot \nabla \boldsymbol{u}+\boldsymbol{u} \cdot \nabla \hat{\boldsymbol{e}}+\hat{\boldsymbol{e}} \times(\nabla \times \boldsymbol{u})+\boldsymbol{u} \times(\nabla \times \hat{\boldsymbol{e}})]=(\nabla \times \boldsymbol{u}) \cdot[\hat{\boldsymbol{e}} \times(\nabla \times \boldsymbol{u})] .
$$

The triple scalar product on the right-hand side vanishes, while the term in brackets on the left-hand side is simply $\nabla(\hat{\boldsymbol{e}} \cdot \boldsymbol{u})$; thus,

$$
(\nabla \times \boldsymbol{u}) \cdot \nabla(\hat{\boldsymbol{e}} \cdot \boldsymbol{u})=0,
$$

i.e. the component of velocity in the direction of zero gradient is constant along vortex-lines.

Note: The hypothesis of the theorem includes all two-dimensional flows and all unidirectional solenoidal flows. The theorem is entirely kinematical; the only restriction placed on the velocity field is differentiability.

\section{REFERENCES}

Aung, W. 1972 Fully developed laminar free convection between vertical plates heated asymmetrically. Intl J. Heat Mass Transfer 15, 1577-1580.

BATCheloR, G. K. 1954 Heat transfer by free convection across a closed cavity between vertical boundaries at different temperatures. Q. Appl. Maths 12, 209-233.

Bejan, A. \& Tien, C. L. 1978 Fully developed natural counterflow in a long horizontal pipe with different end temperatures. Intl J. Heat Mass Transfer 21, 701-708.

Chait, A. \& Korpela, S. A. 1989 The secondary flow and its stability for natural convection in a tall vertical enclosure. J. Fluid Mech. 200, 189-216.

Daniels, P. G. 1985 Transition to the convective regime in a vertical slot. Intl J. Heat Mass Transfer 28, 2071-2077.

DAvis, S. H. 1967 Convection in a box: linear theory. J. Fluid Mech. 30, 465-478.

Dixon, M. \& Probert, S. D. 1975 Heat-transfer regimes in vertical, plane-walled, air-filled cavities. Intl J. Heat Mass Transfer 18, 709-710.

Dryden, H. L., Murnaghan, F. D. \& Bateman, H. 1956 Hydrodynamics. Dover.

ECKERT, E. R. G. \& CARLSON, W. O. 1961 Natural convection in an air layer enclosed between two vertical plates with different temperatures. Intl J. Heat Mass Transfer 2, 106-120.

Elder, J. W. 1965 Laminar free convection in a vertical slot. J. Fluid Mech. 23, 77-98.

Fusegi, T., Hyun, J. M. \& Kuwahara, K. 1991 Three-dimensional simulations of natural convection in a sidewall-heated cube. Intl J. Numer. Meth. Fluids 13, 857-867.

Fusegi, T., Hyun, J. M. \& Kuwahara, K. 1993 Three-dimensional natural convection in a cubical enclosure with walls of finite conductance. Intl J. Heat Mass Transfer 36, 1993-1997.

Gebhart, B., Jaluria, Y., Mahajan, R. L. \& Sammakia, B. 1988 Buoyancy-Induced Flows and Transport, textbook edn. Hemisphere.

Gershuni, G. Z. \& ZhukhovitskiI, E. M. 1976 Convective Stability of Incompressible Fluids (transl. D. Louvish). Keter.

Gill, A. E. \& DaVeY, A. 1969 Instabilities of a buoyancy-driven system. J. Fluid Mech. 35, 775-798.

Girault, V. \& Raviart, P.-A. 1979 Finite Element Approximation of the Navier-Stokes Equations. Springer.

Hart, J. E. 1971 Stability of flow in a differentially heated inclined box. J. Fluid Mech. 47, 547-576. 
Jones, R. C. \& Furry, W. H. 1946 The separation of isotopes by thermal diffusion. Rev. Mod. Phys. 18, 151-224.

Klosse, K. \& Ullersma, P. 1973 Convection in a chemical vapor transport process. J. Crystal Growth 18, 167-174.

LAmB, H. 1932 Hydrodynamics, 6th edn. Cambridge University Press.

LeONG, W. H., Hollands, K. G. T. \& BRunger, A. P. 1998 On a physically-realizable benchmark problem in internal natural convection. Intl J. Heat Mass Transfer 41, 3817-3828.

Leong, W. H., Hollands, K. G. T. \& Brunger, A. P. 1999 Experimental Nusselt numbers for a cubical-cavity benchmark problem in natural convection. Intl J. Heat Mass Transfer 42 , 1979-1989.

McBaIn, G. D. 1998 Heat and mass transfer across tall cavities filled with gas-vapour mixtures: The fully developed regime. Intl J. Heat Mass Transfer 41, 1397-1403.

McBain, G. D. Vapour transport across gas-filled enclosures. PhD thesis, James Cook University, Townsville (in preparation).

Mallinson, G. D. 1987 The effects of side-wall conduction on natural convection in a slot. Trans. ASME C: J. Heat Transfer 109, 419-426.

Mallinson, G. D. \& VAhl Davis, G. DE 1973 The method of the false transient for the solution of coupled elliptic equations. J. Comput. Phys. 12, 435-461.

Mallinson, G. D. \& VAhl Davis, G. DE 1977 Three-dimensional natural convection in a box: A numerical study. J. Fluid Mech. 83, 1-31.

Nagata, M. \& Busse, F. H. 1983 Three-dimensional tertiary motions in a plane shear layer. J. Fluid Mech. 135, 1-26.

Ostroumov, G. A. 1958 Free convection under the conditions of the internal problem. NACA Tech. Mem. 1407.

Raithby, G. D. \& Hollands, K. G. T. 1985 Natural convection. In Handbook of Heat Transfer Fundamentals (ed. W. M. Rohsenhow, J. P. Hartnett \& E. N. Ganić), 2nd edn. McGraw-Hill.

Schladow, S. G., Patterson, J. C. \& Street, R. L. 1989 Transient flow in a side-heated cavity at high Rayleigh number: a numerical study. J. Fluid Mech. 200, 121-148.

Vest, C. M. \& ARPaCI, V. S. 1969 Stability of natural convection in a vertical slot. J. Fluid Mech. 36, $1-15$

Viskanta, R., KIM, D. M. \& GaU, C. 1986 Three-dimensional natural convection heat transfer of a liquid metal in a cavity. Intl J. Heat Mass Transfer 29, 475-485. 